\section{Modelling of thermal hydraulics in a KAROLINA calorimeter for its calibration methodology validation}

\author{
Aleksandra Luks, \\ Krzysztof Pytel, \\ Mikołaj Tarchalski, \\ Nikołaj Uzunow, \\ Tomasz Krok
}

\begin{abstract}
Results of numerical calculations of heat exchange in a nuclear heating detector for nuclear reactors are presented in this paper. The gamma radiation is generated in nuclear reactor during fission and radiative capture reactions as well as radioactive decay of its products. A single-cell calorimeter has been designed for application in the MARIA research reactor in the National Centre for Nuclear Research (NCBJ) in Świerk near Warsaw, Poland, and can also be used in the Jules Horowitz Reactor (JHR), which is under construction in the research centre in Cadarache, France. It consists of a cylindrical sample, which is surrounded by a gas layer, contained in a cylindrical housing. Additional calculations had to be performed before its insertion into the reactor. Within this analysis, modern computational fluid dynamics (CFD) methods have been used for assessing important parameters, for example, mean surface temperature, mean volume temperature, and maximum sample (calorimeter core) temperature. Results of an experiment performed at a dedicated out-of-pile calibration bench and results of numerical modelling validation are also included in this paper.
\end{abstract}

Key words: computional fluid dynamics (CFD) - differential calorimeter $\bullet$ gamma heating $\bullet$ gamma rays $\bullet$ Jules Horowitz Reactor (JHR) • modelling of thermal hydraulics $\bullet$ nuclear heating $\bullet$ nuclear reactor $\bullet$ research reactor MARIA

\author{
A. Luks $\bowtie$ \\ Innovative Nuclear and Sustainable Power Engineering, \\ Institute of Heat Engineering, \\ Warsaw University of Technology, \\ 21/25 Nowowiejska Str., 00-665 Warsaw, Poland, \\ Tel.: +48 60148 2038, \\ E-mail: aluks@itc.pw.edu.pl \\ K. Pytel, M. Tarchalski, T. Krok \\ National Centre for Nuclear Research, \\ 7 Andrzeja Sołtana Str., 05-400 Otwock/Swierk, Poland \\ N. Uzunow \\ Institute of Heat Engineering, \\ Warsaw University of Technology, \\ 21/25 Nowowiejska Str., 00-665 Warsaw, Poland
}

Received: 18 August 2015

Accepted: 26 July 2016

\section{Introduction}

The calorimetric method is one of the well-known methods of measurement of gamma radiation strength in nuclear reactors. It is based on direct measurement of heat deposited by photons within sample owing to photo effect, Compton effect, and pair production. A measurement realized using this method results in a dependence between the supplied heat and the sample temperature. Heating or cooling with constant intensity is the most often applied temperature conditions [1].

A single-cell calorimeter has been designed for application in the MARIA research reactor in the National Centre for Nuclear Research in Świerk near Warsaw, Poland. Not only the results of this elaboration are to be used in further analysis of the MARIA reactor operation but they are also dedicated for Jules Horowitz Reactor (JHR) analysis by the research centre in Cadarache, France. The latter reactor is under construction and will be used widely for science and production of radioisotopes for medical purposes. Because of some similarities between these two reactors, results obtained in MARIA core may also be used for the JHR operation analysis.

The main task of this work is to prove the validity of the new calibration methodology developed for KAROLINA single-cell nuclear heating calorimeter. For that purpose, numerical methods of CFD, 


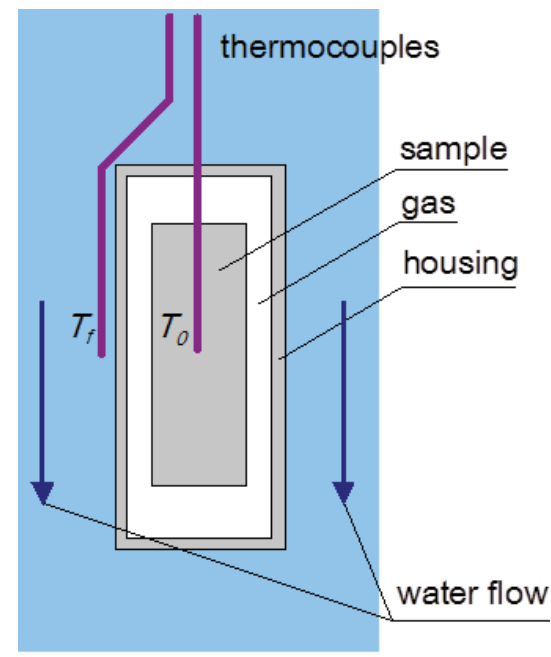

Fig. 1. Schematic cross section of the single-cell calorimeter.

broadly applied for solving such problems, have been used.

\section{Theoretical model of calorimeter}

The calorimeter consists of a cylindrical sample placed on bearers of high thermal resistance, surrounded by a gas layer, contained in a cylindrical housing. The sample material should be of high thermal conductivity. The sample is equipped with temperature sensor, a thermocouple. The cross section of the calorimeter is shown in Fig. 1.

The sample's form determines calorimeter's sensitivity range, which allows measuring of low-intensity or high-intensity nuclear heating. Single-cell construction permits the reduction of thermocouples to two. Inert gas (helium) inside the calorimeter, secured by its tightness, is the main factor of the calorimeter thermal properties. Calorimeter housing, during measurements, is cooled by the calibration bench water.

The single-cell calorimeter is made to measure the nuclear heating in nuclear reactors that consist mainly of gamma radiation. The gamma radiation sources are the ${ }^{235} \mathrm{U}$ fission reactions and next decays of the fission products. The heat generated in the sample is transferred by conduction through the gas and housing layers to the cooling water. The gap between the sample and the housing is 1-mm thick and filled with helium gas under atmospheric pressure. Such a solution minimizes natural convection in the gas layer.

Nuclear heating measurement expressed in watts per gram is basically deduced from the difference in temperature between the sample and the housing.

\section{Analytical study}

The temperature field in the sample is substituted by difference between the sample temperature $T(r, t)$ in determined point $r$ and time $t$ and water temperature $T_{f}$, according to the definition [2]:

$$
\vartheta(r, t)=T(r, t)-T_{f}
$$

The water temperature is assumed to be constant. The temperature field in the form of Eq. (1) corresponds to the equation of heat conduction in transient conditions with internal heat sources:

$$
\rho C \frac{\partial \vartheta(r, t)}{\partial t}=\lambda \nabla^{2} \vartheta(r, t)+q_{v}
$$

complemented by the Fourier boundary condition:

$$
-\left.\lambda \frac{\partial \vartheta}{\partial n}\right|_{S}=\frac{1}{R} \vartheta_{S}
$$

where $\rho$ is the sample density, $C$ the heat capacity, $\lambda$ the body's heat conductivity, $q_{v}$ the internal heat source, and $R$ the heat resistance.

Integrating Eq. (2) over the sample volume and applying the Gauss theorem and boundary condition (3) lead to the following form:

$$
\rho C \frac{d \bar{\vartheta}_{V}}{d t}=-\frac{S}{R V} \bar{\vartheta}_{s}+q_{v}
$$

where $S$ is the cross-sectional area, $V$ is the volume, and $\bar{\vartheta}_{V}$ and $\bar{\vartheta}_{S}$ are the average temperatures, respectively, over the sample volume and sample surface:

$$
\begin{gathered}
\bar{\vartheta}_{V}=\frac{1}{V} \iiint \vartheta d V \\
\bar{\vartheta}_{S}=\frac{1}{S} \iint \vartheta d S
\end{gathered}
$$

The method of coupling the issues of conduction and convection is shown by the following formula:

$$
\begin{aligned}
\rho C \frac{d \bar{\vartheta}_{V}}{d t} & +\rho C \frac{\partial \bar{\vartheta}_{s} \cdot V_{x}}{\partial x}+\rho C \frac{\partial \bar{\vartheta}_{s} \cdot V_{y}}{\partial y} \\
& =-\frac{S}{R V} \bar{\vartheta}_{s}+\rho \cdot g+q_{v}
\end{aligned}
$$

where $g$ is the gravity coefficient.

The temperature of the sample $\vartheta^{*}$ is measured in certain point of the sample, close to its centre. By introducing two temperature non-uniformity coefficients:

$$
\begin{aligned}
& \psi_{V}=\bar{\vartheta}_{V} / \vartheta * \\
& \psi_{S}=\bar{\vartheta}_{S} / \vartheta^{*}
\end{aligned}
$$

one can rewrite Eq. (4) in the following form:

$$
\frac{d \vartheta^{*}}{d t}=-\frac{S \psi_{S}}{R V \rho C \psi_{v}} \vartheta *+\frac{q_{v}}{\rho C \psi_{v}}
$$

When reformulating Eq. (4) to Eq. (10), the hidden assumption has been made, that non-uniformity coefficients are invariable in time. Heat transfer within the sample could be characterized by a dimensionless similarity number, the Biot number (Bi). It is interpreted as the ratio of the heat conduction resistance in a body to the heat convection resistance on its outer surface and described by an appropriate dependence [2]: 


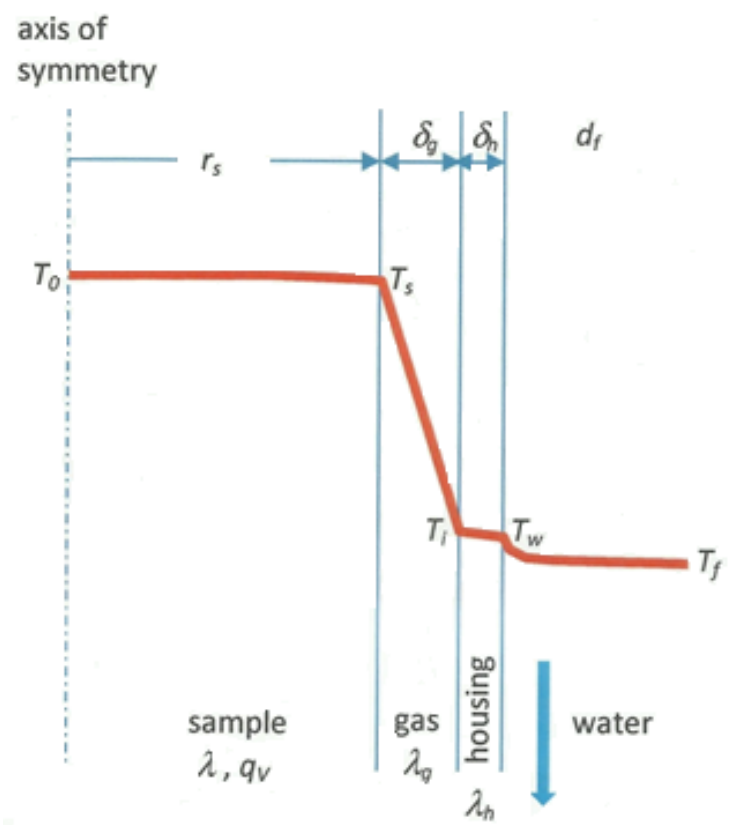

Fig. 2. Radial temperature distribution in the calorimeter.

$$
\mathrm{Bi}=\frac{R_{\lambda}}{R_{\alpha}}=\frac{\alpha \cdot r_{S}}{\lambda}
$$

where $\alpha$ is the heat convection coefficient between the body and the fluid and $\lambda$ the body's heat conductivity.

For low heat conduction resistance of calorimeter sample confronted with high convection resistivity of gas, $\mathrm{Bi} \rightarrow 0(\mathrm{Bi}<0.1)$, the temperature distribution within the sample is even (see schematic temperature profile in Fig. 2) and non-uniformity coefficients are very close to 1 .

It is believed that this is valid for both steady states and transients. The CFD thermal-hydraulic simulation is aimed at the determination of the temperature distribution with given heat source distribution as well as with known ambient coolant heat and flow parameters for steady states and transients.

The solution of Eq. (10) for a calorimeter rapidly inserted into the reactor core with the initial condition of $\vartheta *(0)=0$, which corresponds to a simulation of a water-cooled calorimeter, but without heating, is

$$
\vartheta *(t)=\frac{q_{v}}{\rho C m \psi_{v}}\left(1-e^{-m t}\right)
$$

Having determined the time constant $m$ and heat capacity $C$, one can deliberately use the formula (12) for the determination of gamma heating rate:

$$
q_{V} / \rho=\vartheta *(\infty) \cdot m \cdot C
$$

from saturation temperature in steady-state conditions. Thus, the time constant $m$ can be considered as a calibration constant for the measurements of gamma heating by means of single-cell calorimeter.

The inversed time constant $m$ could be experimentally determined by measuring the changes of $\vartheta^{*}(t)$ in time after the rapid insertion of the calorimeter into the reactor.
It could also be determined during another transient process, such as rapid removal of the calorimeter from the reactor. In this case, an initial condition of $\vartheta^{*}(0)=\vartheta_{\text {heating }}(\infty)$ has to be assumed, which corresponds to a calorimeter in a reactor core in a steady state.

After certain period (to achieve so-called ordered state), the temperature $\vartheta *$ changes exponentially:

$$
\vartheta *(t) \approx e^{-m t}
$$

Ordered state can be characterized by a dimensionless similarity number, the Fourier number (Fo). It is interpreted as the ratio of the heat conduction flux to the internal energy of heat accumulated in the body and described by an appropriate dependence [2]:

$$
\mathrm{Fo}=\frac{a \cdot t}{l^{2}}=\frac{\lambda \cdot t}{C \cdot \rho \cdot l^{2}}
$$

where $a$ is the thermal diffusivity between the body and the fluid and $l$ the characteristic dimension describing the body's geometry. The limit Fourier number for calorimeter is equal to approximately 1206.88 in case of graphite and 328.28 in case of lead.

Similar conditions for measurements of calorimeter time constant provide out-of-core experiment (see section "Experiment at the out-of-core stand"). The calorimeter is heated in a furnace and moved quickly to a cooling bath after achieving temperature stabilization. The temperature $\vartheta *$ response described by Eq. (14) is observed with the same time constant $m$. Such out-of-core measurement may serve as a standard calorimeter calibration procedure.

\section{CFD simulation}

\section{Mesh}

The calorimeter geometry is moderately complex. This imposed specific assumptions in relation to the initial model development and calculation mesh generation process. First of all, a simplified model of the real geometry was applied, in which the bearing and thermocouples visible in Fig. 3 were not taken into account.

The model was discretized for the simulation purposes. A structural mesh consisting of almost 0.6 million quadrangular cells was generated. This has been done using the GAMBIT 2.4.6 software, applying the finite-volume method. The mesh is shown in Fig. 4. It was condensed in the marked boundary layer in order to simulate better the real water flow. The density mesh is very important, and it could influence the results. Therefore, the fine mesh was used in the liquid region $(y+<5)$ to treat the boundary layer. Different regions such as

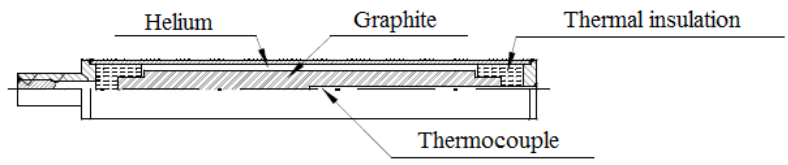

Fig. 3. Cross section of the KAROLINA calorimeter. 


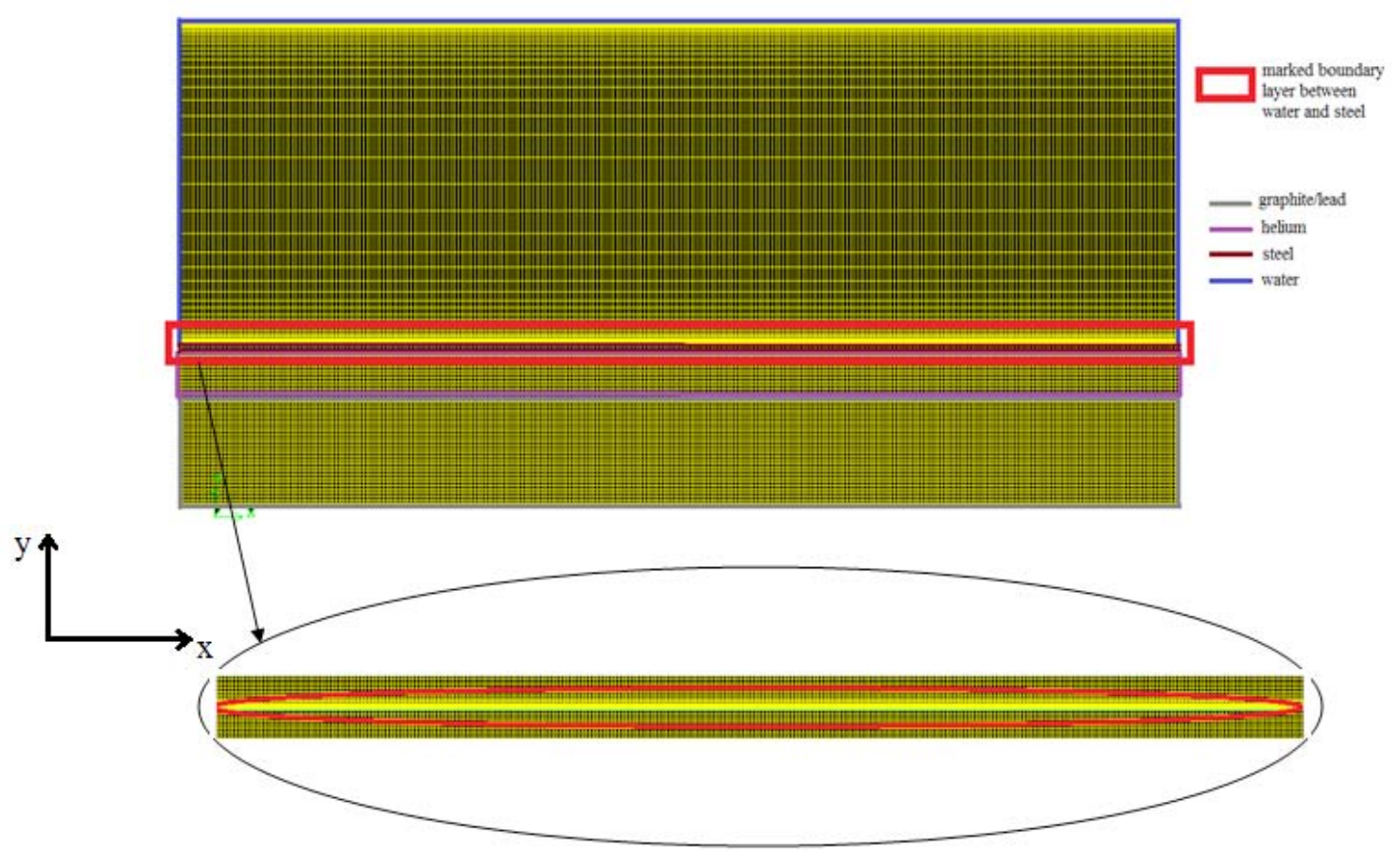

Fig. 4. Model calculation mesh, consisting of 571300 cells.

sample (graphite or lead), gas gap (helium), steel housing, and water are marked in different colours.

There are no skew cells in the mesh. This results from dividing the model into additional volumes. At the same time, this is an advantage, as skew elements often give worse calculation results and may even cause simulation instabilities. The application of quadrangular elements enables better modelling of the flow conditions.

\section{Solver}

The numerical simulations were performed using the ANSYS FLUENT 14.0 environment.

The calorimeter model consists of a cylindrical sample of $6 \mathrm{~mm}$ in diameter, made either of graphite (first case) or lead (second case). It is surrounded by a 1-mm gap filled with helium and contained in a steel housing cooled by flowing water.

Before starting the numerical simulation, the initial and boundary conditions have been determined. They are presented in Table 1.

A two-dimensional model was applied. The axial symmetry allows to assume that results from a three-dimensional model should be similar to those from the two-dimensional one.

The $k-\varepsilon$ realizable model of turbulence was selected. It takes the possibility of flow separation from the wall into account. This phenomenon causes higher flow resistance. In addition, compared to the $k-\varepsilon$ standard or $k-\omega$ turbulence models, it gives a velocity profile closer to the correct ones.

Because of the Reynolds number of $5.7 \times 10^{4}$, the $k-\varepsilon$ realizable turbulence model was applied. The boundary conditions for the $k-\varepsilon$ equations (a system of two differential equations) are as follows: - turbulence intensity, for a developed flow, 4\%; - turbulence characteristic dimension, $0.018 \mathrm{~m}$.
With thermal-hydraulic parameters, given in Table 2, and a power density of $5 \mathrm{~W} / \mathrm{g}$, the graphite sample reaches a maximum temperature of 396.7 K, while the increase in water temperature is only about $1 \mathrm{~K}$. When graphite is replaced by lead, higher temperatures are observed, and this is a more serious challenge in terms of thermal limitations, as the melting temperature of lead equals $600.65 \mathrm{~K}$. A lower value of the volumetric power density has been selected in order the sample temperature to not exceed the melting temperature of lead.

With thermal-hydraulic parameters, given in Table 2, and a power density of $1 \mathrm{~W} / \mathrm{g}$, the lead sample reaches a maximum temperature of $427.17 \mathrm{~K}$. The applied numerical model is presented in Table 3.

\section{Results and comments}

\section{Results}

Three most interesting temperatures were selected in the mathematical model: the sample average surface temperature, the sample average volumetric temperature, and the sample maximum temperature. The changes in maximum temperatures of graphite

Table 1. Initial and boundary conditions in the model

\begin{tabular}{lc}
\hline \multicolumn{1}{c}{ Condition } & Type of condition \\
\hline Inlet & Water inlet velocity: $2.1 \mathrm{~m} / \mathrm{s}$ \\
Outlet & Temperature: $313.15 \mathrm{~K}$ \\
Walls & Pressure outlet \\
Axis & Wall material: steel \\
Solid zone & Symmetry in axis of the calorimeter \\
Fluid zone & Graphite/Lead \\
Operating & Water (liquid) $/$ Helium \\
conditions & Operating temperature: $313.15 \mathrm{~K}$ \\
\hline
\end{tabular}


Table 2. Summary of thermal-hydraulic parameters for the materials used in the model

\begin{tabular}{|c|c|c|c|c|c|}
\hline \multirow{2}{*}{ Parameter } & \multicolumn{5}{|c|}{ Material } \\
\hline & Water & Steel & Helium & Graphite & Lead \\
\hline Velocity [m/s] & 2.1 & - & - & - & - \\
\hline Hydraulic diameter [m] & 0.018 & - & - & - & - \\
\hline Kinematic viscosity $\left[\mathrm{m}^{2} / \mathrm{s}\right]$ & $6.59 \mathrm{E}-7$ & - & - & - & - \\
\hline Thermal conductivity $[\mathrm{W} /(\mathrm{m} \cdot \mathrm{K})]$ & 0.63 & 19.1 & 0.1792 & 115 & 34.9 \\
\hline Dynamic viscosity $[\mathrm{kg} /(\mathrm{m} \cdot \mathrm{s})]$ & $6.53 \mathrm{E}-6$ & - & $1.701 \mathrm{E}-5$ & - & - \\
\hline Specific heat $[\mathrm{J} /(\mathrm{kg} \cdot \mathrm{K})]$ & 4173.26 & 461 & 5204 & 754 & 125 \\
\hline Density $\left[\mathrm{kg} / \mathrm{m}^{3}\right]$ & 992.26 & 7860 & 0.1305 & 1685 & 11340 \\
\hline Temperature $[\mathrm{K}]$ & 313.15 & - & - & - & - \\
\hline Radius of core $[\mathrm{m}]$ & - & - & - & 0.003 & 0.003 \\
\hline Thickness [m] & - & 0.0007 & 0.001 & - & - \\
\hline
\end{tabular}

Table 3. Introduction of computer simulation model

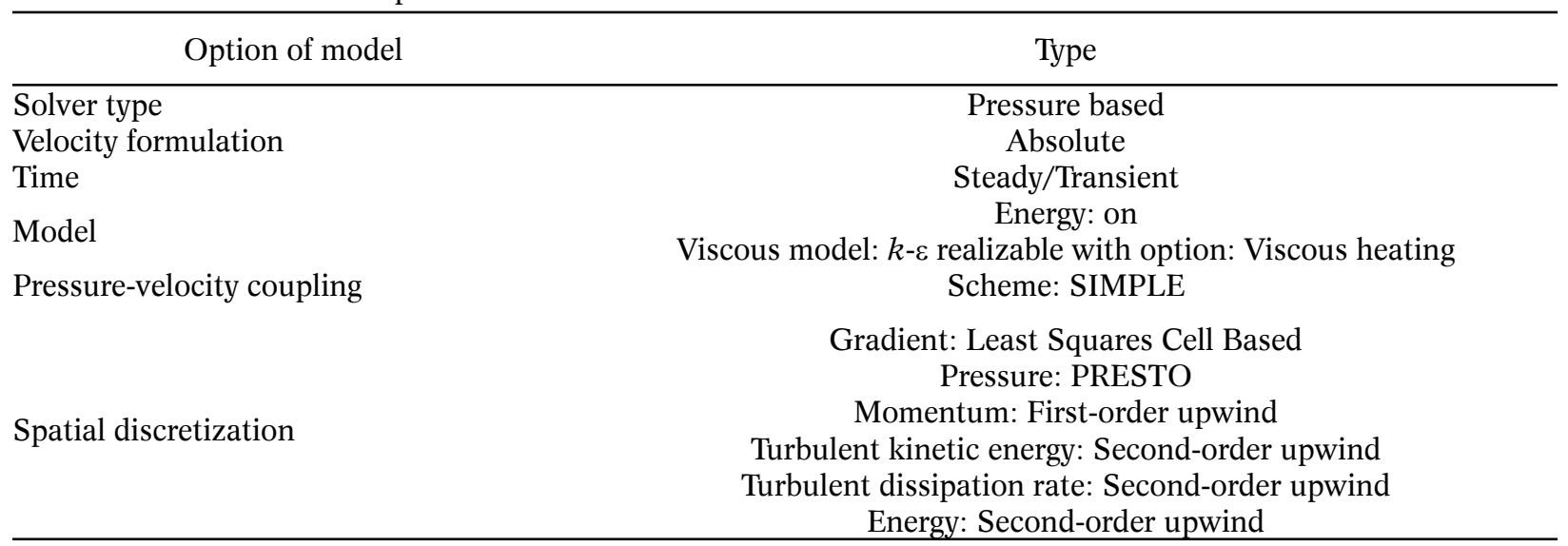

and lead are shown in Fig. 5. The time of obtaining a steady state equals to about 2 min.

For the transient calorimeter heating, an exponential curve has been fitted to the difference between the maximum temperature of the sample and surface temperature, given by the dependence $\sim(1-\exp (-m \cdot t))$, which confirms the analytical considerations.

For graphite, the reversed time constant $m$ equals $0.0797 \mathrm{~s}^{-1}$ and the temperature difference $\vartheta^{*}(\infty)$ during the process is equal to $83.3 \mathrm{~K}$. The main temperature drop appears in the gas layer and equals $80.8 \mathrm{~K}$. Assuming the specific heat for graphite from Table 2 , one can determine the heat generation from Eq. (12), $q_{V} / \rho=5.005 \mathrm{~W} / \mathrm{g}$, that is, exactly the same as that assumed in the heating calculations. The temperature non-uniformity coefficients have values close to 1 , that is, $\psi_{s} \geq 0.9918$ and $\psi_{v} \geq 0.9959$.

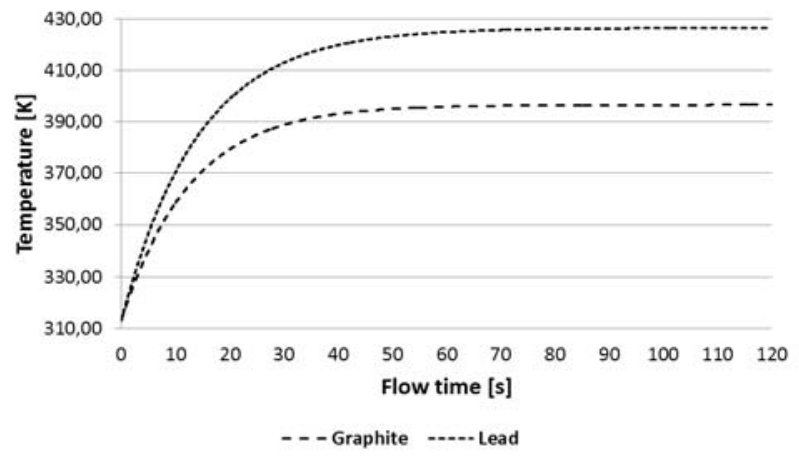

Fig. 5. Time course of the increase in maximum temperature of graphite and lead while heating the calorimeter.
For lead, the reversed time constant $m$ equals $0.0705 \mathrm{~s}^{-1}$ and the temperature differences $\vartheta^{*}(\infty)$ during the process was equal to $113.82 \mathrm{~K}$ with the main temperature drop on the gas layer equals to $110.32 \mathrm{~K}$. Taking specific heat for lead from Table 2, one can determine the heat generation from Eq. (12) $q_{V} / \rho=1.003 \mathrm{~W} / \mathrm{g}$, again exactly the same as that assumed in the heating calculations. The temperature non-uniformity coefficients also have values close to 1 , that is, $\psi_{s} \geq 0.9775$ and $\psi_{v} \geq 0.9887$. Those values are not close to 1 as in the case of graphite because of the (worse) thermal parameters of lead.

The temperature distribution in graphite and lead calorimeters is shown in Figs. 6 and 7, respectively.

The processes of cooling of graphite/lead calorimeters have been simulated for two cases:

- in-core case, that is, rapid removal of calorimeter from the reactor core, and

- out-of-core case, that is, rapid removal of calorimeter from the furnace.

In case of in-core cooling, the initial and boundary conditions were assumed, corresponding to the steady-state heating simulation. For the out-of-core case, the isothermal conditions for entire calorimeter were assumed with temperature equal to the average sample temperature from steady-state heating simulation.

The time course of changes in sample temperature are shown in Figs. 8 and 9 for graphite and lead calorimeter, respectively. In each figure, the in-core cooling is marked in blue and the one concerning the out-of-core cooling is marked in red. In both the cases, the curves corresponding to in-core and 


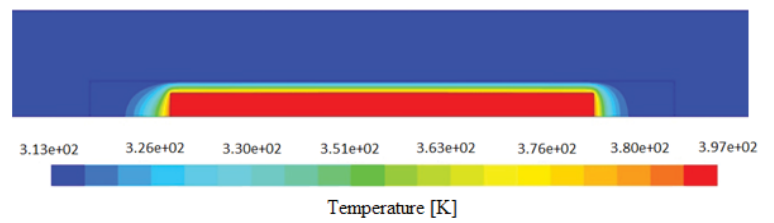

Fig. 6. Temperature field in the graphite calorimeter.

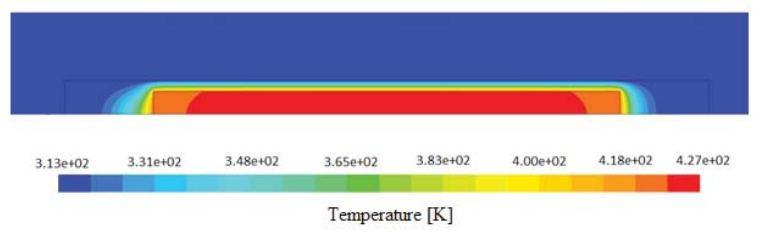

Fig. 7. Temperature field in the lead calorimeter.

out-of-core cooling overlap, which means that results of transient calorimeter measurements at the out-of-core stand would be identical to those in reactor cooling conditions. The curves are exponential, described by the dependence $\sim \exp (-m \cdot t)$.

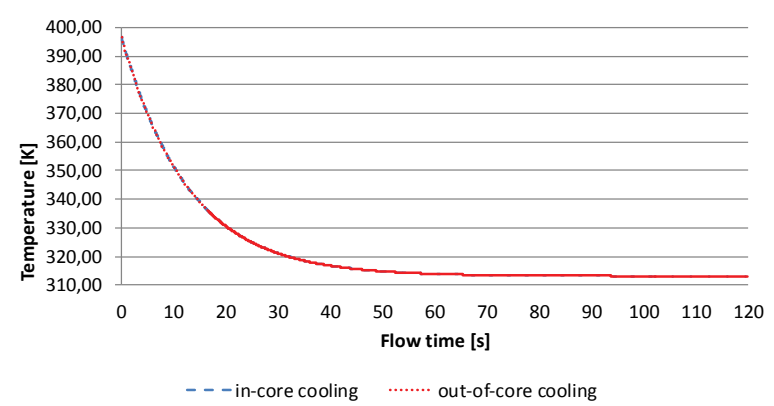

Fig. 8. Change in the temperature of the graphite calorimeter after sudden removal of the detector from a reactor and cooling at an out-of-core stand.

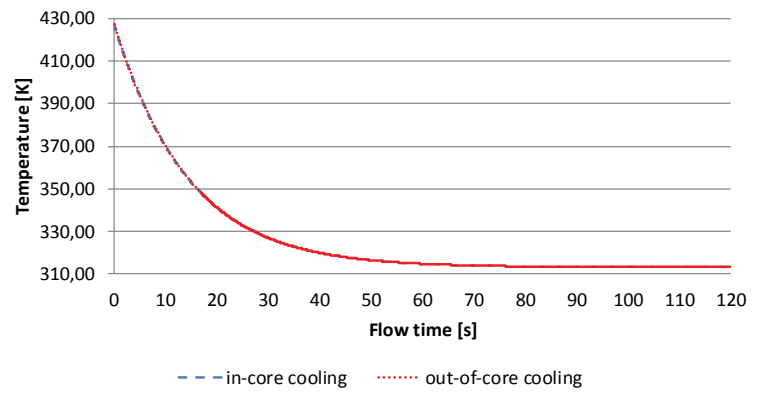

Fig. 9. Change in the temperature of the lead calorimeter after sudden removal of the detector from a reactor and cooling at an out-of-core stand.

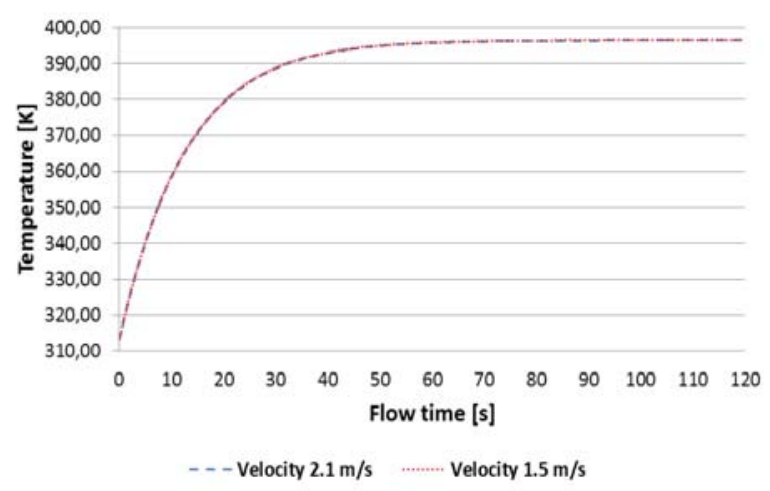

Fig. 10. Effect of the flow velocity on the temperature inside the calorimeter.

\section{Numerical model study}

The numerical model study comprised, first, the effect of cooling water flow velocity on the calorimeter temperatures. Next, the two actions concern the influence of the time interval and the number of nodes of the calculation mesh on the temperature values obtained in the model.

The single-cell gamma calorimeter was designed for measuring the nuclear heating (mostly gamma) in the MARIA reactor irradiation channels. Each channel has a different water flow velocity. It depends on the channel diameter, its position, concomitant devices, and general reactor core configuration. The exact water flow velocity within the particular channels is not determined precisely. One could base only on the water pressure drop measurements for the whole reactor core. Therefore, the effect of coolant velocity on the calorimeter's thermal behaviour needs to be determined. The calculation results show no effect of the flow velocity on the temperatures achieved inside the calorimeter. In Fig. 10, the exponential curve of the temperature for velocity of $2.1 \mathrm{~m} / \mathrm{s}$ is marked in blue, and for velocity of $1.5 \mathrm{~m} / \mathrm{s}$, it is marked in red. The two curves ideally coincide.

The Courant-Friedrichs-Levy (CFL) condition is applied for determining the proper time step $(\Delta t)$ for the numerical solution [3]. It is used for the stability and convergence analysis of numerical methods of solution of time-dependent problems, described by differential equations. It is applied for equations describing advection, such as the Navier-Stokes equation. Thanks to the CFL condition, the value of the time step is determined. This value cannot exceed the result of the following dependence, as the method would be unstable:

$$
\frac{V_{x} \cdot \Delta t}{\Delta x}+\frac{V_{y} \cdot \Delta t}{\Delta y} \leq C
$$

The constant $C$ is called non-dimensional Courant number. In practical applications, it usually equals 1 . In Eq. (16), velocities in $x$-direction and $y$-direction are mentioned by symbols $V_{x}$ and $V_{y}$.

In order to reduce the time and computing power consumption, the calculation time step was increased up to $0.01 \mathrm{~s}$. The results for two radically different time steps, shown in Fig. 11, do not differ. This justifies the application of longer time step.

Compression of the calculation grid provides more accurate result information. However, this also increases the calculation time consumption.

Three different grids were applied in the model. A distance of 0.0001 between the nodes corresponds to 571300 elements; with a distance of 0.0002 , there are 237778 elements; and for a distance of 0.001 , 232288 elements are produced. The performed calculations show that there are no differences between the obtained results.

The exponential curves, shown in Fig. 12, ideally coincide. This justifies the application of longer distance between the grid nodes, which would provide smaller number of elements and reduction of the calculation time. 


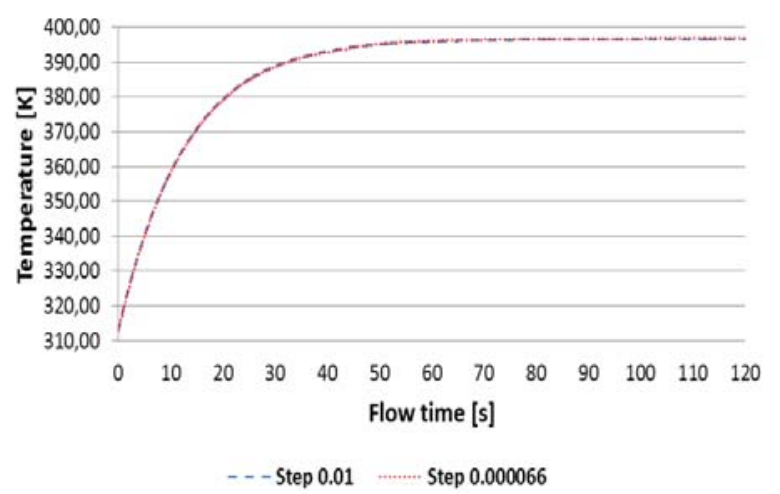

Fig. 11. Comparison of temperatures for different time steps.

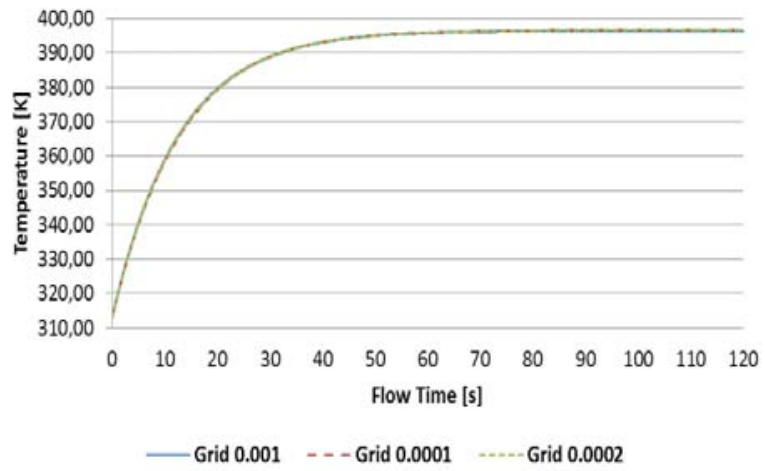

Fig. 12. Comparison of temperatures for different numbers of grid elements.

\section{Experiment at the out-of-core stand}

The main element of out-of-core stand is a furnace, to which the differential calorimeter is introduced from above. There the calorimeter is heated up to $160^{\circ} \mathrm{C}$ until temperature equilibrium state. Next it is moved to a vessel in which it is being cooled by pumped water in similar way as it could be done in the reactor pool. During the experiment, the water flow rate was at the level of $39-40.21 / \mathrm{min}$, that is, about $2.4 \mathrm{~m}^{3} / \mathrm{h}$. The measuring stand is shown in Fig. 13 .

The time course of temperature changes during the experiment is shown in Fig. 14. After certain time, the internal (inside the calorimeter) and external (in the furnace) temperatures become equal. Next, after moving the calorimeter to the vessel, it cools down to water temperature, which is around $20^{\circ} \mathrm{C}$. An important experiment feature is its repeatability.

The reversed time constant $m$ turned out to be in fact not constant during the experiment. This is due to the temperature dependence of thermal parameters of graphite and predominantly of helium. The dependence of $m$ parameter on the temperature of helium is shown in Fig. 15. The red line is the linear fit to the results, and such linear relationship is further used in gamma heating measurements.

\section{Conclusions}

This aim of this work was to numerically verify the main features of single-cell calorimeter with special emphasis paid on equivalency of its in-core and out-of-core calibration.

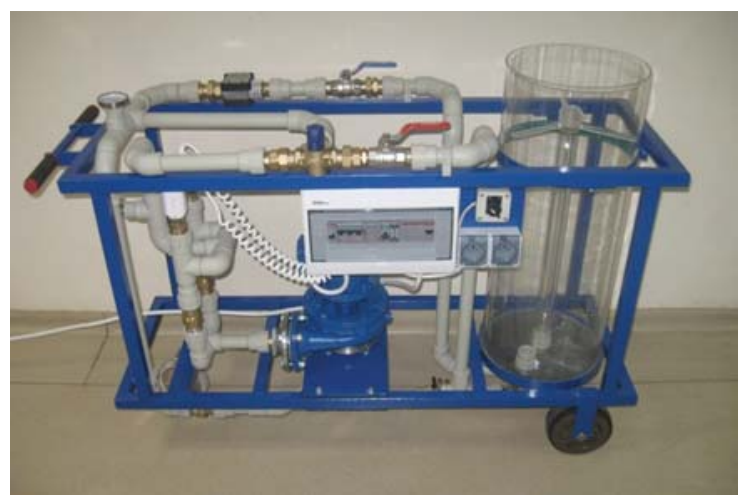

Fig. 13. The out-of-core stand.

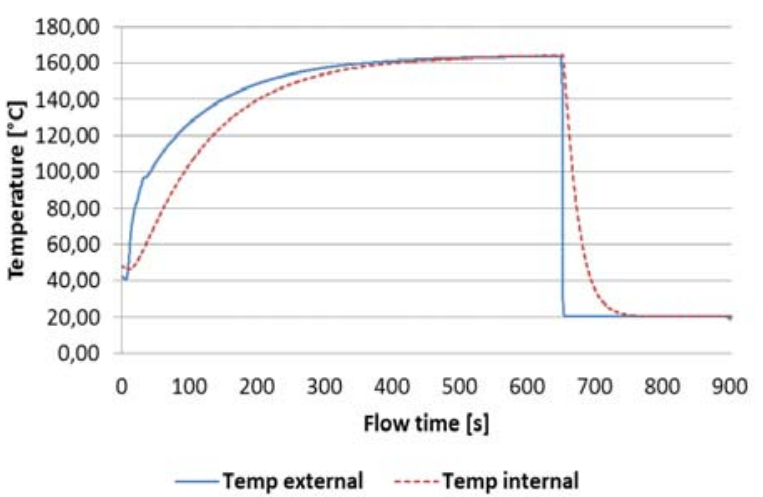

Fig. 14. Temperature changes during heating and cooling the calorimeter.

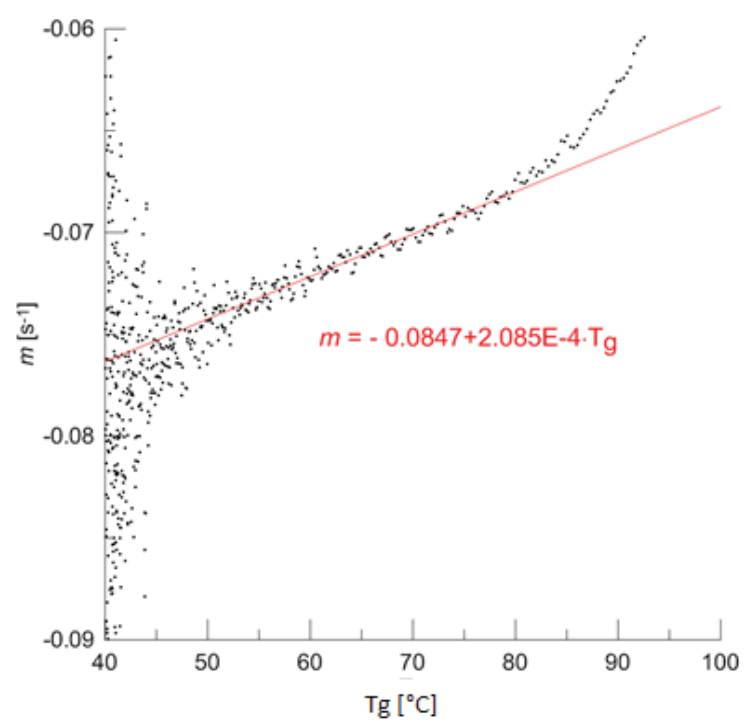

Fig. 15. Dependence of the reversed time constant on the temperature of helium.

Mathematical model of the calorimeter, recalled in section "Theoretical model of calorimeter", uses an assumption that the temperature distribution within the calorimeter sample is very even in both steady-state and transient conditions. This has been fully confirmed by the CFD simulations.

The numerical simulations of transient (both heating or cooling) demonstrated that calorimeter has exponential response to step change of heat generation and the resulting time constant can be used as a calibration constant for the determination of gamma heating in steady states (Eq. (13)). 
Besides, numerical simulation revealed full equivalence of calibration of calorimeter during in-core and out-of-core cooling transients.

Taking advantage of numerical model of calorimeter, the effect of cooling water flow velocity on calorimeter response was investigated; proving that in a wide range of velocities, this effect can be neglected.

Some research on optimization of calculation costs has been performed, concerning the grid density and the time step during transients. This should be useful in further works with simulation of such type detectors. It has been also checked that two-dimensional axially symmetrical model of calorimeter gives practically the same results as the three-dimensional one.

Two different materials of the calorimeter sample were used in the model: graphite and lead. The first can be used for heat generation measurement in the middle of a reactor core, while the lead calorimeter - because of its thermal properties and sensitivity can be used only in a reactor core periphery.

This project should be treated as an intermediate step in a wider research process. Further analyses of different types of calorimeters are planned, especially concerning material properties. Experiments with single-cell calorimeter, performed at the out- -of-core stand, apparently showed that the gaseous helium thermal properties need to be profoundly investigated.

Recently, the experiment with the single-cell calorimeter has been performed at MARIA reactor in Swierk (Poland) and similar measurements are foreseen at the JHR facility in Cadarache (France).

Successful CFD modelling of single-cell calorimeter and comparison with experiments, both in-core and out-of-core, gave rise to further research on different types of calorimeter and another gamma heating measuring detector - a gamma thermometer.

Acknowledgments. The authors of this paper wish to thank Mr. Adam Małkiewicz for sharing photos of the out-of-core stand.

\section{References}

1. Kiełkiewicz, M. (1990). Pomiary w reaktorach jadrowych. Warszawa: WNT.

2. Wiśniewski, S., \& Wiśniewski, T. S. (2009). Wymiana ciepta. Warszawa: WNT.

3. ANSYS Inc. (2011). ANSYS fluent theory guide. Release 14.0. Southpointe: ANSYS, Inc. 